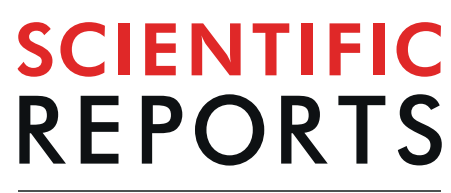

natureresearch

Check for updates

\title{
OPEN Reverse genetics reveals single gene of every candidate on Hybrid sterility, XChromosome QTL 2 (Hstx2) are dispensable for spermatogenesis
}

Kento Morimoto ${ }^{1}$, Koki Numata ${ }^{2,3}$, Yoko Daitoku ${ }^{4}$, Yuko Hamada ${ }^{4}$, Keiko Kobayashi ${ }^{4,5}$, Kanako Kato ${ }^{4}$, Hayate Suzuki ${ }^{6}$, Shinya Ayabe ${ }^{7}$, AtsushiYoshiki ${ }^{7}$, Satoru Takahashi ${ }^{4}$, Kazuya Murata ${ }^{4}$, Seiya Mizuno ${ }^{4 凶}$ \& Fumihiro Sugiyama ${ }^{4}$

F1 hybrid progenies between related subspecies often show hybrid sterility (HS) or inviability. HS is caused by failure of meiotic chromosome synapsis and sex body formation in house mouse. Previous studies identified two HS critical genomic regions named Hst 2 on $\mathrm{Chr} X$ and $H s t 1$ on $\mathrm{Chr} 17$ by murine forward genetic approaches. HS gene on Hst1 was reported to be Prdm9. Intersubspecific polymorphisms of Prdm9 induce HS in hybrids, and Prdm9 null mutation leads to sterility in the inbred strain. However, HS gene on Hstx2 remains unknown. Here, using knock-out studies, we showed that $\mathrm{HS}$ candidate genes on $\mathrm{Hst} \times 2$ are not individually essential for spermatogenesis in B6 strain. We examined 12 genes on Hstx2: Ctag2, 4930447F04Rik, Mir743, Mir465d, Mir465c-2, Mir465b-1, Mir465c-1, Mir465, Gm1140, Gm14692, 4933436I01Rik, and Gm6812. These genes were expressed in adult testes, and showed intersubspecific polymorphisms on expressed regions. This first reverse genetic approach to identify HS gene on $\mathrm{Hst} 2 \mathrm{2}$ suggested that the loss of function of any one HS candidate gene does not cause complete sterility, unlike Prdm9. Thus, the mechanism(s) of HS by the HS gene on Hstx2 might be different from that of Prdm9.

Postzygotic reproductive isolation between related subspecies often results in hybrid incompatibility such as hybrid sterility (HS) or inviability ${ }^{1-4}$. This HS is the barrier distinguishing related species by disturbing the gametogenesis in the hybrid. HS is widely observed around intersubspecific hybrids such as yeast, plants, insects, birds, and mammals ${ }^{1,2,4}$. In 1922, Haldane's rule was reported as the observation that if only one sex of the hybrid is inviable or sterile, that sex is the heterogametic sex (XY or ZW $)^{5}$. Additionally more than 80 years ago, Dobzhansky, T. (1937) and Muller, H. (1942) proposed a two-locus model called Dobzhansky-Muller incompatibility (hereafter DMI) hypothesis that postzygotic isolation arises from a negative epistatic interaction between incompatible alleles that evolved on two independent evolutionary lineages ${ }^{6,7}$. Previous studies showed a large effect of Chr $\mathrm{X}(\mathrm{Chr} \mathrm{Z})$ on reproductive isolation, particularly on HS or hybrid inviability ${ }^{8-11}$. In the last 30 years, several HS genes and hybrid inviability genes were identified in budding yeast, thale cress, fruit fly, and house mouse ${ }^{12-25}$. In Drosophila, HS genes: $\mathrm{OdsH}$ and $\mathrm{O} v d$, and hybrid inviability gene: $\mathrm{Hmr}$ and locate on $\mathrm{Chr} \mathrm{X}^{13,14,16,21}$. However in

${ }^{1}$ Laboratory Animal Science, Doctoral Program in Medical Sciences, University of Tsukuba, 1-1-1 Tennodai, Tsukuba, Ibaraki, 305-8575, Japan. 'Laboratory Animal Science, Bachelor of Medical Science, University of Tsukuba, 1-1-1 Tennodai, Tsukuba, Ibaraki, 305-8575, Japan. ${ }^{3}$ Department of Clinical Laboratories, University of Tsukuba Hospital, 2-1-1 Amakubo Tsukuba, Ibaraki, 305-8576, Japan. ${ }^{4}$ Laboratory Animal Resource Center and Trans-border Medical Research Center, Faculty of Medicine, University of Tsukuba, 1-1-1 Tennodai, Tsukuba, Ibaraki, 305-8575, Japan. ${ }^{5}$ Developmental Engineering \& Embryology Group Genetically Engineered Models and Services Charles River Laboratories Japan, Inc., 955 Kamibayashi, Ishioka, Ibaraki, 315-0138, Japan. ' Laboratory Animal Science, Doctoral Program in Biomedical Sciences, University of Tsukuba, 1-1-1 Tennodai, Tsukuba, Ibaraki, 305-8575, Japan. ${ }^{7}$ Experimental Animal Division, RIKEN BioResource Research Center, 3-1-1 Koyadai, Tsukuba, Ibaraki, 305-0074, Japan. ${ }^{凶}$-mail: konezumi@md.tsukuba.ac.jp 
house mice, these orthologous genes have not reported. Although several researchers proposed to explain this heterogametic HS or inviability as being controlled by Chr X (Chr Z) in vertebrata ${ }^{26-28}$, the X-linked (Z-linked) causative gene for Haldane's rule and DMI has remained unclear for over a century.

The two HS loci, Hst 1 and Hstx2, were found by murine forward genetics ${ }^{29-32}$. The first HS locus in mice, Hst1 was identified as a polymorphic variant on Chr 17 between two laboratory strains, C57BL10/Sn and C6H/Di, both predominantly of Mus musculus domesticus (hereafter Mmd) origin ${ }^{29}$. To identify HS gene on Hst1, Forejt, J. et al. established HS murine recombinant inbred model using $\mathrm{PWD} / \mathrm{Ph}$ (hereafter PWD) inbred strain derived from Mus musculus musculus (hereafter $\mathrm{Mmm}$ ) subspecies and C57BL/6 (hereafter B6) inbred strain derived from $M m d$ subspecies ${ }^{33,34}$. PWD was established from a single pair of wild mice of the $M m m$ subspecies caught in 1972 in Central Bohemia, Czech Republic ${ }^{35}$. Both subspecies diverged from a common ancestor approximately 0.3 to 0.5 million years ago ${ }^{36}$. This model shows asymmetric HS, wherein $(\mathrm{PWD} \times \mathrm{B} 6)$ F1 hybrid (mating PWD female with B6 male) shows sterility, while $(\mathrm{B} 6 \times$ PWD) F1 hybrid (mating B6 female with PWD male) shows semi-fertility ${ }^{35,36}$. To identify these HS loci, Gregorová, S. et al. also established intersubspecific chromosome substitution (consomic) strains $\mathrm{C} 57 \mathrm{BL} / 6-\mathrm{Chr} \#^{\mathrm{PWD} / \mathrm{Ph}} / \mathrm{Fore}$ J carrying individual $\mathrm{Mmm}$ chromosomes or their parts on $M m d$ background ${ }^{37}$. The HS gene on Hst 1 was identified by the forward genetic approach as PR domain containing $9(\operatorname{Prdm} 9)^{24}$, and later was shown to control meiotic recombination hotspots ${ }^{38,39}$. Prdm 9 encodes for histone $\mathrm{H} 3$ lysine 4 trimethyltransferase that controls the hotspots of DNA double strand breaks (DSBs) at meiotic prophase in spermatocytes. Hstx2 was found out by consomic strains C57BL/6-Chr X ${ }^{\mathrm{PWD} / \mathrm{Ph}} / \mathrm{ForeJ}$ carrying $\mathrm{Mmm}$ Chr X or their parts on $M m d$ background ${ }^{32}$. The $4.7 \mathrm{Mb}$ (Chr X:64.9 Mb-69.6 Mb, GRCm38) HS critical region on Chr X, Hstx2, was identified by quantitative trait locus (QTL) analysis of male fertility phenotypes in these consomic strains ${ }^{32}$. They revealed that HS is also controlled by Hstx 2 of PWD PW $^{32}$

Unlike the HS gene Prdm 9 on Hst1, the HS gene on Hstx2 remains unknown. Identifying the molecular mechanism of HS controlled by Chr X can unravel the molecular mechanism of Haldane's rule in vertebrata. Forward genetic approaches have a limit to narrow down to only $4.7 \mathrm{Mb} H s t x 2$ critical region, because Hstx 2 may lie on a recombination cold spot ${ }^{40,41}$. Hence, reverse genetic approach is required to identify the HS gene on Hstx2.

In a mouse model of intersubspecific hybrids between B6 and PWD, HS needs three factors, Prdm 9 het-

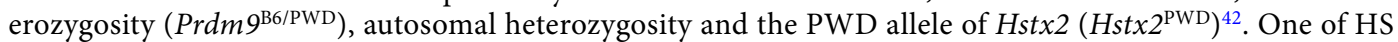
mechanism model was explained by hybrid incompatibility between $\operatorname{Prdm} 9^{\mathrm{B} 6 / \mathrm{PWD}}$ and autosomal heterozygosity without DMI at least in house mouse ${ }^{43}$. Minisatellite structure of the zinc finger array coding on DNA binding sites of PRDM9 has interspecific polymorphisms between $\mathrm{B} 6$ and $\mathrm{PWD}^{43,44}$. The binding sites were evolutionally eroded by DNA double-strand breaks determined by PRDM9 in each strain ${ }^{43,44}$. The affinity of PRDM9 $9^{\mathrm{B} 6}$ to B6 autosomes and the affinity of PRDM9 ${ }^{\mathrm{PWD}}$ to PWD autosomes decreases with evolution, respectively ${ }^{43,44}$. This is because the evolutionary erosion of genomic DNA occurs independently in each mouse sub-strain. In hybrid, PRDM9 ${ }^{\mathrm{B} 6}$-determined hotspots occur mostly on the PWD chromosome and vice vers $a^{43,44}$. The major meiotic consequences of asymmetric DSB hotspots result in unrepaired DNA DSBs, meiotic asynapsis and abnormal sex body formation, leading to disruption of sperm formation ${ }^{43}$. Nevertheless, only this model cannot explain the asymmetric HS because this meiotic arrest is also controlled by Hstx2. Hst $x 2^{\mathrm{PWD}}$ is responsible for full sterility of $(\mathrm{PWD} \times \mathrm{B} 6) \mathrm{F} 1$ hybrid, while the B6 allele of $H s t \times 2\left(H s t \times 2^{\mathrm{B} 6}\right)$ attenuates fertility of $(\mathrm{B} 6 \times \mathrm{PWD}) \mathrm{F} 1$ hybrid $^{32}$. However, how Hstx2 regulates HS remains elusive. Therefore, we proposed three hypotheses of the role of $H s t \times 2$. First hypothesis is that the spermatogenic function of HS gene(s) on Hstx2 is lost in (PWD $\times$ B6) F1 male but partially remains in $(\mathrm{B} 6 \times \mathrm{PWD}) \mathrm{F} 1$ male. Second hypothesis is that dominant-negative $H s t \times 2^{\mathrm{PWD}}$ impedes the spermatogenesis in hybrids. Third hypothesis is that $H s t x 2^{\mathrm{B} 6}$ rescues fertility of hybrids. Here, to verify whether Hst 2 are essential for spermatogenesis in the first hypothesis, we aimed to delete HS gene on Hstx2 in B6 mice. We hypothesised that HS gene on Hstx2 may have similar features to $\operatorname{Prdm} 9$, such as expressing in adult testes $^{45}$, having intersubspecific polymorphisms on expressed region between $\mathrm{Mmm}$ and $\mathrm{Mmd} \mathrm{d}^{43,44}$, explaining HS mechanism by single locus ${ }^{43}$ and inducing sterility by knock-out $(\mathrm{KO})$ in B6 genetic background ${ }^{45,46}$. If our first hypothesis is correct, the HS gene on Hstx2 KO B6 mice would be sterile. Therefore, we aimed to identify HS gene on Hstx2 through KO study.

To identify HS candidate gene, we investigated the expression of 32 genes on Hstx2 in adult B6 testes using databases. Next, we analysed intersubspecific polymorphisms on expressed regions between B6 and PWD, also PWK/Phj (Mmm inbred strain, hereafter, PWK) by past reports and public database.

\section{Results}

Twelve HS candidate genes were identified on Hstx2. Male sterility of reciprocal hybrids between PWD and B6 is controlled by Hstx2 (64.9 Mb-69.6 Mb, GRCm38) $)^{32}$, which carries 10 protein-coding genes and 22 microRNA (miRNA) genes (Fig. 1). As mentioned earlier, if our hypothesis is correct, the HS gene on Hstx2 KO B6 mice must be sterile. To find out the HS gene from the 32 genes on Hstx2, we firstly screened them for expression in adult B6 testes. Testes expression was analysed using Fantom5 (http://fantom.gsc.riken.jp/5/), BioGPS (http://biogps.org/circadian/\#goto=welcome) and miRbase (http://www.mirbase.org/), which revealed that of the 32 genes, 9 protein-coding genes: Ctag2, 4930447F04Rik, Gm1140, Gm14692, 4933436101Rik, Fmr1, Fmr1nb, Gm6812, and Aff2, and all 22 miRNA genes were expressed in adult B6 testes (Table 1 and S1). The only one gene, Slitrk2 showed no expression in adult B6 testes (Table 1). Next, we analysed intersubspecific polymorphism of the genes on Hstx2. We focused on intersubspecific polymorphism on expressed regions, which are the coding region in protein-coding alleles and mature miRNA region in miRNA alleles. Bhattacharyya et al. reported intersubspecific missense single nucleotide polymorphisms (SNPs) of HS candidate genes among B6, PWD, and $\mathrm{PWK}^{32}$ (Table 1). PWK is a Mmm inbred strain and closely related to $\mathrm{PWD}^{47}$. By comparison of the B6 allele of HS candidate genes with that of the PWD allele, Ctag2, 4930447F04Rik, Slitrk2, Mir743, 4933436I01Rik, Fmr1nb, and Aff2 were found to carry missense SNPs between B6 and PWD. Fmr1 does not carry any intersubspecific missense SNPs. We excluded Slitrk2, Fmr1, Fmr1nb, and Aff2 on Hstx2 as the targets, because these genes have 


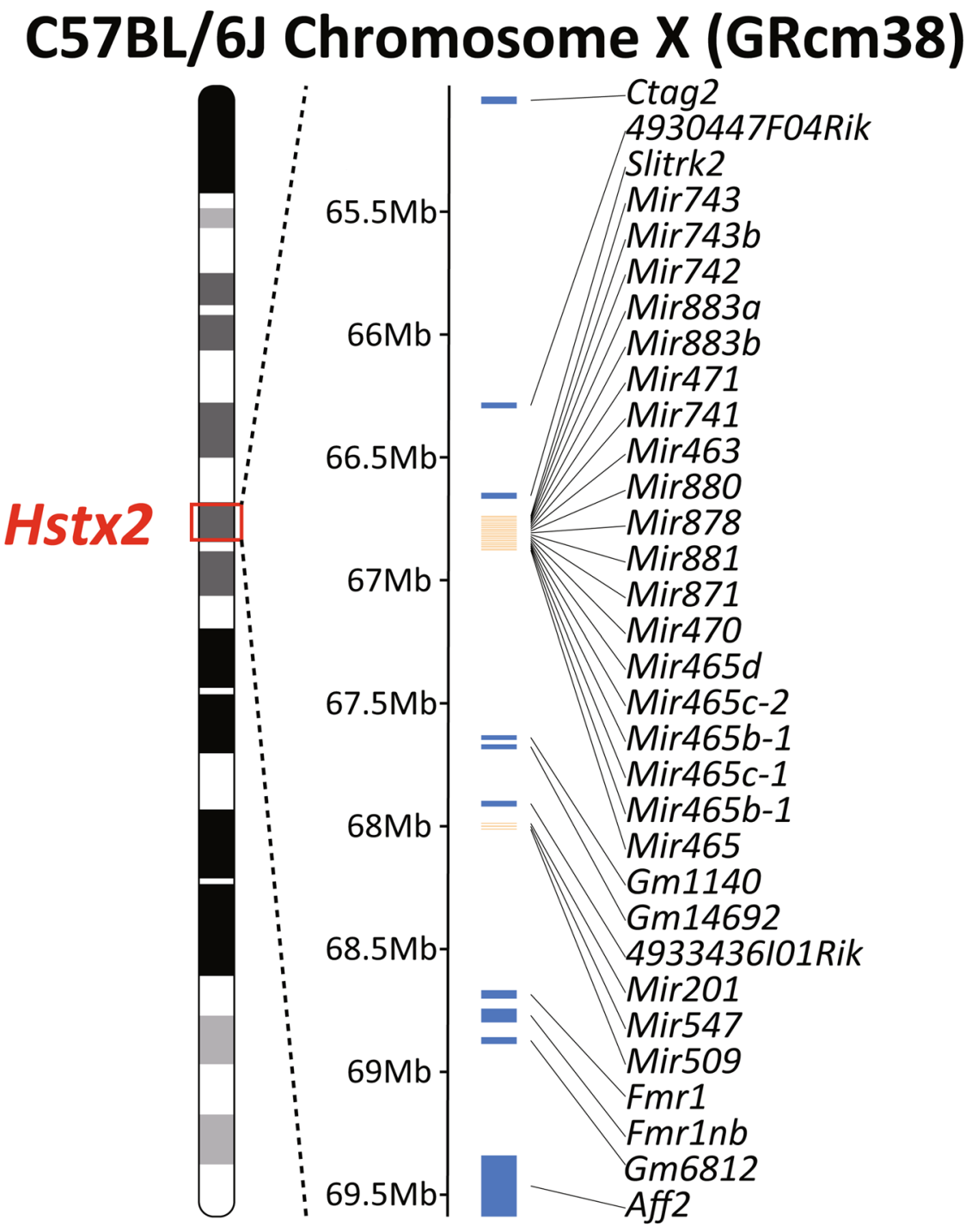

Figure 1. Gene map of the Hstx2 critical region on Chr X. Gene map of the Hstx2 critical region on Chr X (64.9Mb-69.6 Mb, GRCm38). Hstx2 carries 10 protein-coding genes and 22 miRNA genes.

already been reported as not being necessary for spermatogenesis in B6 male by KO studies ${ }^{48-51}$. Additionally, we targeted Gm1140, Gm14692, Gm6812, and Mir465 cluster because Gm1140, Gm14692, Gm6812, Mir465c-2, Mir465b-1, Mir465c-1, and Mir465 also have intersubspecific missense SNPs between B6 and PWK (Table S1). The next generation sequence (NGS) data on PWK was obtained from Sanger Institute Mouse Genome Project (http://www.sanger.ac.uk/science/data/mouse-genomes-project) and we aligned PWK and B6 reference genome (GRCm38). A very recent study reported that $G m 1140$, Gm14692, and Gm6812 have intersubspecific missense SNPs between B6 and PWD, and Mir465 cluster shows copy number polymorphism between B6 and PWD (and PWK $)^{40}$. Therefore, we added these genes to the targets.

Taken together, we targeted 12 HS candidate genes, composed of six protein-coding genes, namely Ctag2, 4930447F04Rik, Gm1140, Gm14692, 4933436I01Rik, and Gm6812, and six miRNA genes, namely Mir743, Mir465d, Mir465c-2, Mir465b-1, Mir465c-1, and Mir465.

Generation of eight HS candidate gene/cluster KO male mice by CRISPR/Cas9 system. To investigate whether each of the $12 \mathrm{HS}$ candidate genes are essential for spermatogenesis, we generated each HS candidate gene KO mice using CRISPR/Cas9 system. We respectively designed two single guide RNA (sgRNA) sites, one upstream of the target gene and the other downstream of it on B6 genetic background in order to remove all genomic region. Mir465d, Mir465c-2, Mir465b-1, Mir465c-1, and Mir465 are located on Mir465 cluster, which is composed of 6 repeats (Fig. S1); as we could not design sgRNA for each gene of the Mir465 cluster, we decided to knock out the complete Mir465 cluster. To create each of the eight HS candidate gene/cluster KO mice, we performed the microinjection of $p X 330$, the circular plasmid carrying sgRNA and Cas 9 expression units, or the electroporation of sgRNA and Cas9 nuclease into B6 mouse eggs gained by mating with B6 male or in vitro fertilisation (IVF), and then each $\mathrm{KO} \mathrm{F} 0$ founder were selected for $\mathrm{KO}$ allele, removing all genomic 


\begin{tabular}{|l|l|l|l|l|l|}
\hline Gene & $\begin{array}{l}\text { testis } \\
\text { expression }\end{array}$ & $\begin{array}{l}\text { SNP } \\
\text { position }\end{array}$ & B6 & PWD & PWK \\
\hline Ctag2 & + & $65,047,953$ & T & G & G \\
\hline 4930447F04Rik & + & $66,303,564$ & A & C & C \\
\hline Slitrk2 & - & $66,655,874$ & A & G & G \\
\hline & & $66,656,111$ & A & G & G \\
\hline Mir743 & + & $66,776,774$ & T & C & C \\
\hline 493436101 Rik & + & $67,920,137$ & C & T & T \\
\hline & & $67,920,143$ & T & G & G \\
\hline & & $67,920,312$ & G & T & T \\
\hline & & $67,920,431$ & T & A & A \\
\hline & & $67,920,805$ & T & A & A \\
\hline & & $67,920,818$ & C & T & T \\
\hline & & $67,920,822$ & T & G & G \\
\hline Fmr1 & + & - & - & - & - \\
\hline Fmr1nb & + & $68,762,025$ & C & G & G \\
\hline & & $68,769,064$ & T & A & A \\
\hline Aff2 & + & $69,544,913$ & A & G & G \\
\hline & & $69,830,745$ & C & T & T \\
\hline & & $69,830,760$ & C & G & G \\
\hline & & $69,830,782$ & A & T & T \\
\hline & & $69,834,780$ & G & A & A \\
\hline
\end{tabular}

Table 1. Testis expression and intersubspecific missense SNPs between B6 and PWD of candidate genes on Hstx2.

\begin{tabular}{|c|c|c|c|c|c|c|c|c|c|}
\hline \multirow[b]{2}{*}{ Gene } & \multicolumn{2}{|c|}{\begin{tabular}{|l|} 
Number of embryos \\
\end{tabular}} & \multicolumn{3}{|c|}{ Number of pups } & \multicolumn{4}{|c|}{ Number of founders } \\
\hline & Injected & Transferred $^{\mathrm{a}}$ & Newborn ${ }^{b}$ & Weaning ${ }^{\mathrm{c}}$ & Birthrate $^{b / a}$ & Male & Female & Total $^{\mathrm{d}}$ & $\begin{array}{l}\text { Mutation } \\
\text { efficiency }{ }^{d / c}\end{array}$ \\
\hline Ctag2 & 83 & 80 & 31 & 31 & $38.8 \%$ & 13 & 8 & 21 & $67.7 \%$ \\
\hline 4930447F04Rik & 84 & 82 & 27 & 27 & $32.9 \%$ & 13 & 7 & 20 & $74.1 \%$ \\
\hline Mir743 & 155 & 146 & 41 & 38 & $28.1 \%$ & 7 & 7 & 14 & $36.8 \%$ \\
\hline Mir465 cluster & 75 & 72 & 26 & 24 & $36.1 \%$ & 4 & 3 & 7 & $29.2 \%$ \\
\hline$G m 1140$ & 266 & 249 & 38 & 36 & $15.3 \%$ & 2 & 1 & 3 & $8.33 \%$ \\
\hline Gm14692 & 100 & 98 & 18 & 16 & $18.4 \%$ & 1 & 2 & 3 & $18.8 \%$ \\
\hline 4933436I01Rik & 105 & 90 & 32 & 31 & $35.6 \%$ & 10 & 7 & 17 & $54.8 \%$ \\
\hline Gm6812 & 112 & 90 & 6 & 6 & $6.67 \%$ & 1 & 2 & 3 & $50.0 \%$ \\
\hline
\end{tabular}

Table 2. Generation of each the HS candidate gene KO founders by CRISPR/Cas9 system.

region of target gene. To confirm the CRISPR/Cas9 mutations, we performed genotyping using genomic PCR from mouse tail and then confirmed the $\mathrm{KO}$ allele. Thus, we generated each of the eight gene/cluster $\mathrm{KO}$ male founders (Table 2).

Fertility and spermatogenesis of the eight HS candidate gene/cluster KO male mice. To investigate fertility of each candidate gene KO male founder, the sexually mature KO male F0 founder was mated with sexually mature B6 wild-type female. F0 founder have the possibility of mosaicism; therefore, these KO F0 male founder may not show full knock out of the gene in the testes. If $\mathrm{KO}$ allele was inherited to next generation, it would imply that the germ cell carried the KO allele and could be developed to fertilisable sperm. From mating test, each heterozygote KO female mice was born (Table 3). One of $4933496 I 01$ Rik KO male founder generated wild-type female, suggesting that the founder was mosaicism. Except for the above, all other F1 female mice that were born from mating F0 male founders with female B6, showed the KO allele. Therefore, in our experiment, the male founders would not have shown mosaicism. These results suggested that the fertilisable sperm could have developed from the germ cell carrying the $\mathrm{KO}$ allele. Therefore, all eight $\mathrm{KO}$ male genotypes, $1 \mathrm{HS}$ candidate gene/cluster per each, were fertile.

To investigate the spermatogenesis of each $\mathrm{KO}$ mice, histochemical analysis was performed. Peanut agglutinin (PNA) lectin histochemistry (LHC) is available as a marker of sperm. Immunohistochemistry (IHC) of phosphorylated form of the histone variant $\mathrm{H} 2 \mathrm{AX}(\gamma \mathrm{H} 2 \mathrm{AX})$ is a marker of sex body on pachytene spermatocyte. IHC and PNA-LHC of testis section of each KO F0 founder showed sperm and sex body formation on these spermatocytes (Fig. 2). These results suggested that the eight candidate genes/cluster were not individually necessary for sperm formation at least in $\mathrm{B} 6$ genetic background. 


\begin{tabular}{|l|l|l|l|l|l|l|l|}
\hline \multirow{2}{*}{ Gene } & \multirow{3}{*}{$\begin{array}{l}\text { Founder } \\
\text { number }\end{array}$} & Hemizygote & $\begin{array}{l}\text { Wild- } \\
\text { Type }\end{array}$ & Total & Heterozygote & $\begin{array}{l}\text { Wild- } \\
\text { Type }\end{array}$ & Total \\
\cline { 3 - 9 } & $\# 2$ & 0 & 4 & 4 & 6 & 0 & 6 \\
\hline Ctag2 & $\# 3$ & 0 & 8 & 8 & 7 & 0 & 7 \\
\hline & $\# 17$ & 0 & 7 & 7 & 2 & 0 & 2 \\
\hline & $\# 9$ & 0 & 1 & 1 & 1 & 0 & 1 \\
\hline 4930447F04Rik & $\# 9$ & 2 & 2 & 3 & 0 & 3 \\
\hline & $\# 11$ & 0 & 5 & 5 & 2 & 0 & 2 \\
\hline Mir743 & $\# 4$ & 0 & 5 & 5 & 3 & 0 & 3 \\
\hline Mir465 cluster & $\# 1$ & 0 & 6 & 6 & 0 & 0 & 0 \\
\hline & $\# 14$ & 0 & 2 & 2 & 1 & 0 & 1 \\
\hline & $\# 16$ & 0 & 3 & 3 & 3 & 0 & 6 \\
\hline Gm1140 & $\# 8$ & 0 & 8 & 8 & 9 & 0 & 9 \\
\hline Gm14692 & $\# 46$ & 0 & 7 & 7 & 5 & 0 & 5 \\
\hline 4933436101 Rik & $\# 7$ & 0 & 6 & 6 & 4 & 0 & 4 \\
\hline & $\# 8$ & 0 & 8 & 8 & 3 & 5 & 8 \\
\hline & $\# 20$ & 0 & 4 & 4 & 9 & 0 & 9 \\
\hline Gm6812 & $\# 6$ & 0 & & & & & \\
\hline
\end{tabular}

Table 3. Fertility of each the HS candidate gene KO male founders.

PNA/ $/$ H2AX/DAPI
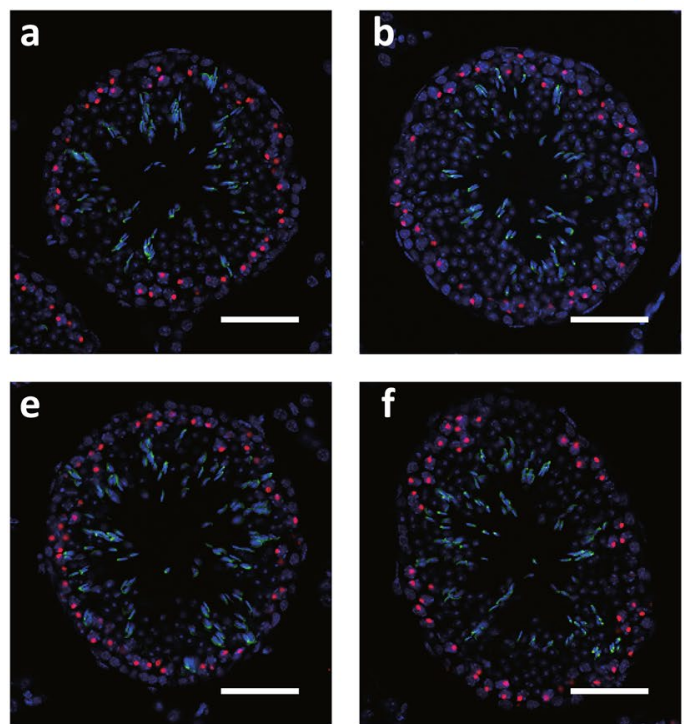
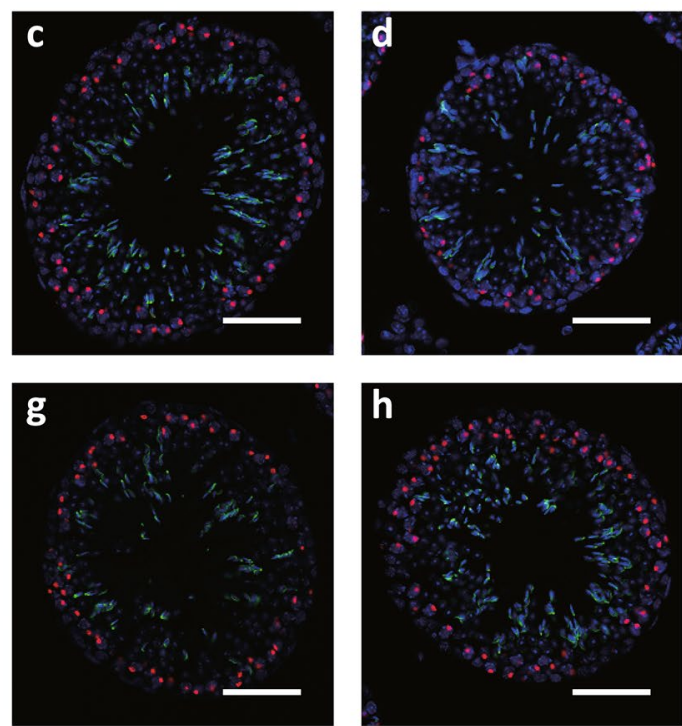

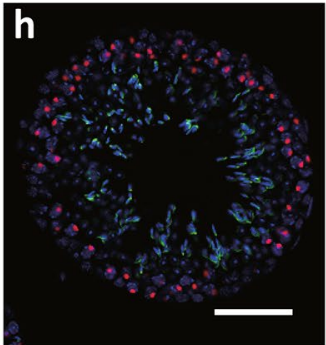

Figure 2. Histochemical analysis of the testis from each of the eight KO founders. PNA-LHC (green), $\gamma \mathrm{H} 2 \mathrm{AX}$ (red), and DAPI (blue) staining of the testis section of Ctag2 (a), 4930447F04Rik (b), Mir743 (c), Mir465 cluster (d), Gm1140 (e), Gm14692 (f), $4933436 I 01 R i k(\mathbf{g})$, and Gm6812 (h) KO founders. All show sperm in lumen of seminiferous tubule and sex body formation. Scale bar: $50 \mu \mathrm{m}$.

\section{Discussion}

Our reverse genetic approach revealed that the eight HS candidates, comprising six protein-coding genes, one miRNA gene and one miRNA cluster, were not individually necessary for spermatogenesis in B6 mice. These results exclude the possibility that the loss of function of one HS gene/cluster on Hstx2 causes sterility, at least in B6 genetic background. In hybrid context between $\mathrm{B} 6$ and PWD, Hst $x 2^{\mathrm{B} 6}$ has semi-fertility but Hst $x 2^{\mathrm{PWD}}$ fully lacks fertility ${ }^{32}$. Therefore, we hypothesised that $H s t \times 2^{\mathrm{B} 6}$ and $H s t \times 2^{\mathrm{PWD}}$ are essential for spermatogenesis in both inbred strains and hybrids, and then the function of $H s t \times 2^{\mathrm{B} 6}$ remains while that of $H s t \times 2^{\mathrm{PWD}}$ is disrupted in the hybrids. While we confirmed whether each HS candidate KO caused severe meiotic defects and meiotic failure in B6 mice, we did not assess more subtle phenotypes, such as a mere reduction in sperm count or motility. Therefore, these HS candidate KO males might show semi-fertility.

Previous studies identified two HS loci by forward genetic approaches using consomic strains ${ }^{29,30}$, and the HS gene on $H s t 1$ was identified as $\operatorname{Prdm} 9^{24}$. However, these forward genetic approaches have a limit to narrow down 
4.7 Mb (Chr X:64.9 Mb-69.6 Mb, GRCm38) of Hstx2 critical region on PWD Chr X, because there may be recombination cold spot on $H s t x 2^{40,41}$. Recently, Lustyk, D. et al. reduced $4.7 \mathrm{Mb}$ region of $H s t x 2$ to $2.7 \mathrm{Mb}$ (Chr X:66.51 $\mathrm{Mb}-69.21 \mathrm{Mb}$ ) by backcross where SPO11-driven Cas 9 nuclease was targeted by CRISPR to Hstx2 interval in female meiotic prophase ${ }^{40}$. Nevertheless, this approach could not identify HS gene on Hstx2. Therefore, a reverse genetic approach is required to identify HS gene on Hstx2.

$\operatorname{Prdm} 9$ is the only identified HS gene and is responsible for sterility phenotypes in male hybrids of house mouse ${ }^{38,39,52}$. Intersubspecific polymorphisms on the minisatellite cording for zinc-finger array of $\operatorname{Prdm} 9$, such as repeat variants and missense SNPs, and autosomal heterozygosity induce asymmetric DNA DSBs that cause sterility in hybrids $s^{43,44}$. Additionally, $\operatorname{Prdm} 9$ is expressed in adult testes and its null mutation leads to sterility in the inbred strain ${ }^{45,46}$. Therefore, we hypothesised that HS gene on Hstx2 may have similar features to $\operatorname{Prdm} 9$ : intersubspecific polymorphisms of HS gene on Hstx2 might cause HS, and the HS gene null mutation may lead to sterility on B6 background, as in the case of $\operatorname{Prdm} 9$. However, some hybrid with a sufficiently high recombination rate did not show sterility by $\operatorname{Prdm} 9$ null mutation ${ }^{46}$, so that $\operatorname{Prdm} 9$ is not essential for meiosis in male mice of every species. At least in inbred male, $\operatorname{Prdm} 9$ is essential for spermatogenesis. In this study, we disregarded intersubspecific polymorphisms on intergenic and intron region. Therefore, another gene on Hstx 2 which has other polymorphisms such as SNPs on its regulatory region might be HS gene. Additionally, we added 4 genes/cluster: Gm1140, Gm14692, Gm6812, and Mir465 cluster to the targets because these genes have intersubspecific missense SNPs between B6 and PWK. A very recent study reported that Gm1140, Gm14692 and Gm6812 have intersubspecific missense SNPs between B6 and PWD, and Mir465 cluster have copy number polymorphism between B6 and $\mathrm{PWD}^{40}$. Therefore, this report supports our selecting.

However, in our study, none of the HS candidate genes on Hstx2 was found to be essential for spermatogenesis on B6 genetic background. In our introduction, we proposed three hypotheses for the role of Hstx2. Based on our findings, we could partially exclude our first hypothesis, which was that the spermatogenic function of HS gene(s) on Hstx2 is lost in $(\mathrm{PWD} \times \mathrm{B} 6) \mathrm{F} 1$ male, but partially remains in $(\mathrm{B} 6 \times \mathrm{PWD}) \mathrm{F} 1$ male. However, in this hypothesis, the polygenic effect is retained. On Hstx2, there are a few related and adjacent genes. The coding sequence of Gm6812 is similar to that of $1700020 N 15 R i k$, which is located nearby Hstx2 (Chr X:69.9 Mb). The coding sequence of Gm6812 has only one missense SNP compared with that of $1700020 N 15 R i k$ in both B6 and PWD mice. The coding sequence of $G m 1140$ is also same as that of $G m 14692$. These genes might complement each other functionally. Besides these possibilities, recent study reported that $\mathrm{KO}$ of several genes on Hstx2 resulted in arrested spermatogenesis in B6 mice ${ }^{53}$. The deletion of each Mir741, Mir871, and Mir880 did not affect spermatogenesis, but deletion of 6 miRNAs, Mir741, Mir463, Mir880, Mir878, and Mir871 disrupted spermatogenesis in B6 mice. Therefore, Mir463 or Mir878 might be essential for spermatogenesis, or polygene of these 6 miRNAs may have a critical role in spermatogenesis. The second hypothesis was that dominant-negative $H s t x 2^{\mathrm{PWD}}$ impedes the spermatogenesis in hybrids. Nineteen miRNAs on Hstx2 BAC transgenic mice carrying these autosomal genes show sterility ${ }^{54}$. Normally, these miRNAs are actively transcribed in spermatogonia and suppressed by meiotic sex chromosome inactivation in pachytene spermatocytes. In this BAC transgenic mice, these miRNAs are mis-expressed on pachytene, which induces spermatogenic defects in the BAC transgenic mice. Among the 19 miRNA genes, Mir743 and the Mir465 cluster showed intersubspecific polymorphisms between B6 and PWD. In our study, we generated Mir743 single gene KO B6 mice and Mir465 cluster KO B6 mice, which did not show male sterility. If the second hypothesis was correct, HS gene KO on Hstx2 of PWD in $(\mathrm{PWD} \times \mathrm{B} 6) \mathrm{F} 1$ male would not show sterility. The third hypothesis was that $H s t x 2^{\mathrm{B} 6}$ rescues fertility of hybrids, while $H s t x 2^{\mathrm{PWD}}$ cannot rescue HS. $(\mathrm{PWD} \times \mathrm{B} 6) \mathrm{F} 1$ show sterility caused by asymmetric DSBs ${ }^{43}$. Although the amount of asymmetric DSB in $(\mathrm{B} 6 \times \mathrm{PWD}) \mathrm{F} 1$ male spermatocyte is equivalent to that in $(\mathrm{PWD} \times \mathrm{B} 6) \mathrm{F} 1$ male, $(\mathrm{B} 6 \times \mathrm{PWD}) \mathrm{F} 1$ male shows semi-fertility ${ }^{43}$. This result suggests that HS in $(\mathrm{B} 6 \times \mathrm{PWD}) \mathrm{F} 1$ male is rescued without recovering the asymmetry of meiotic DSBs. Therefore, we propose another recovery mechanism of HS without repairing asymmetry of meiotic DSBs. We suspect that HS gene of PWD on Hstx2 cannot rescue HS in (PWD $\times$ B6) F1 male, while HS gene of B6 on Hstx 2 can rescue HS in (B6 $\times$ PWD) F1 male, inducing asymmetric HS. If the third hypothesis was correct, HS gene KO on Hstx2 of B6 in (B6 × PWD) F1 male would not show sterility.

In conclusion, we used a new approach to determine HS gene on Hstx2 by reverse genetics, which revealed that Ctag2, 4930447F04Rik, Mir743, Mir465 cluster, Gm1140, Gm14692, 4933436101Rik, and Gm6812 are not individually necessary for spermatogenesis in B6 mice. This result partially proved that the loss of function of one gene/cluster on Hstx2 does not cause complete HS. Furthermore, the HS gene on Hstx 2 might cause HS by different mechanisms than that shown by $\operatorname{Prdm} 9$. Thus, our study contributes toward determination of the HS gene on Hstx 2 by at least excluding out one possibility.

\section{Methods}

Mice. C57BL/6 J (B6) and Jcl:CD1 (ICR) inbred strain were purchased from Charles River Laboratories (Yokohama, Japan) and CLEA Japan (Tokyo, Japan). All mice were housed in plastic cages under pathogen-free conditions in a room maintained at $23.5^{\circ} \mathrm{C} \pm 2.5^{\circ} \mathrm{C}$ and $52.5 \% \pm 12.5 \%$ relative humidity under a 14 -h light: 10 -h dark cycle. Mice had free access to commercial chow (MF; Oriental Yeast, Tokyo, Japan) and filtered water. Animal experiments were carried out in a humane manner with approval from the Institutional Animal Experiment Committee of the University of Tsukuba in accordance with the Regulations for Animal Experiments of the University of Tsukuba and Fundamental Guidelines for Proper Conduct of Animal Experiments and Related Activities in Academic Research Institutions under the jurisdiction of the Ministry of Education, Culture, Sports, Science, and Technology of Japan.

Immunohistochemistry of $\gamma \mathrm{H} 2 \mathrm{AX}$ and PNA lectin histochemistry. All mice were sacrificed over 10 -week-old. After intraperitoneal (i.p.) injection of a fatal dose of pentobarbital, mice were transcardially perfused with phosphate-buffered saline (PBS) under anaesthesia. Testes, from which tunica albuginea were 
removed, were fixed overnight in 10\%-Formaldehyde Neutral Buffer Solution (Nacalai Tesque, Kyoto, Japan) and then dehydrated in $70 \%$ ethanol at room temperature over 1 week. The fixed testes were embedded in paraffin and stored at room temperature until use. The testes were sectioned at a thickness of $6 \mu \mathrm{m}$, deparaffinised, immersed in $0.25 \%$ Triton X-100 in PBS and then the antigens were activated in Target Retrieval Solution (Dako, Santa Clara) for $10 \mathrm{~min}$ at $121^{\circ} \mathrm{C}$. After blocking the sections by the blocking solution $[0.1 \%$ bovine serum albumin (BSA), $0.01 \%$ Tween $20,10 \%$ goat serum in PBS] for $60 \mathrm{~min}$ at room temperature, the sections were incubated with mouse IgG anti- $\gamma \mathrm{H} 2 \mathrm{AX}$ antibody (1:100, \#05-636; Millipore, MA) overnight at $4{ }^{\circ} \mathrm{C}$ in a chamber with high humidity. After washing the sections twice using PBS, goat anti-mouse IgG Alexa Fluor 647 (1:200, \#A28181; Invitrogen, CA) and PNA lectin (1:100, \#L7381-1MG; SIGMA-ALDRICH, MO) were applied on the sections and incubated for $60 \mathrm{~min}$ at room temperature in a dark chamber with high humidity. The sections were washed twice with PBS and incubated with 4,6-diamidino-2-phenylindole (DAPI) in PBS (1:500) for 10 min. After washing the sections using PBS, the sections were mounted with Prolong Gold antifade reagent with DAPI (Invitrogen). Images of seminiferous tubules were captured using a fluorescence microscope CCD camera (\#BZ-X710; KEYENCE, Osaka, Japan) and processed using BZ-X analyzer software (KEYENCE).

Sequencing and genotyping. Genomic DNA was extracted from the tails of 3-week-old mice. Genotyping PCR was performed using PrimeSTAR GXL DNA Polymerase (Takara Bio, Shiga, Japan) or AmpliTaq Gold DNA Polymerase (Applied Biosystems); the primers used for this are listed in Table S2.

Generation of KO mice using CRISPR/Cas9 system. To remove all genomic region of each HS candidate gene, we respectively designed two sgRNAs, one whose target site was upstream of the target gene and the other was downstream of it on B6 genetic background. The sequences of the sgRNAs are listed in Table S3. To create $\mathrm{KO}$ mice, we performed microinjection or electroporation. The microinjection was performed according to previously reported method, with some modification ${ }^{55}$. Female B6 mice were i.p. injected with pregnant mare serum gonadotropin (PMSG) and human chorionic gonadotropin (hCG) with a 48-h interval, and mated with male B6 mice. The zygotes were collected from oviducts. Then, two $p X 330$ (circular, $5 \mathrm{ng} / \mu \mathrm{l}$ each), targeting two CRISPR sites of each HS candidate gene, was injected into the pronuclei according to standard protocols. The injected embryos (pronuclei stage) were then transferred into pseudopregnant ICR females. The electroporation was performed as reported previously, with some modification ${ }^{56}$. The sgRNA was synthesised and purified using the GeneArt Precision gRNA Synthesis Kit (Thermo Fisher Scientific, MA) and dissolved in Opti-MEM (Thermo Fisher Scientific). PMSG and hCG were i.p. injected into female B6 mice with 48-h interval, and unfertilised oocytes were collected from their oviducts. We then performed in vitro fertilisation with these oocytes and sperm from B6 mice according to standard protocols. Five hours later, two sgRNA ( $25 \mathrm{ng} / \mu \mathrm{l}$ each) targeting two CRISPR sites of each HS candidate gene, and GeneArt Platinum Cas 9 Nuclease ( $100 \mathrm{ng} / \mu \mathrm{l}$, Thermo Fisher Scientific) were electroporated into these zygotes using the NEPA 21 electroporator (Nepa Gene, Chiba, Japan). The poring pulse was set to: $225 \mathrm{~V}, 2 \mathrm{~ms}$ pulse width, $50 \mathrm{~ms}$ pulse interval, and +4 pulse number. The transfer pulse was set to: $20 \mathrm{~V}, 50 \mathrm{~ms}$ pulse width, $50 \mathrm{~ms}$ pulse interval, and \pm 5 pulse number (attenuation rate was set to $40 \%$ ). After electroporation, the developed 2-cell embryos were transferred into the oviducts of pseudopregnant ICR females.

Received: 4 December 2019; Accepted: 11 May 2020;

Published online: 03 June 2020

\section{References}

1. Turelli, M. \& Moyle, L. C. Asymmetric postmating isolation: Darwin's corollary to Haldane's rule. Genetics 176(2), 1059-1088, https://doi.org/10.1534/genetics.106.065979 (2007).

2. Good, J. M., Handel, M. A. \& Nachman, M. W. Asymmetry and polymorphism of hybrid male sterility during the early stages of speciation in house mice. Evolution 62(1), 50-65, https://doi.org/10.1111/j.1558-5646.2007.00257.x (2008).

3. Brideau, N. J. \& Barbash, D. A. Functional conservation of the Drosophila hybrid incompatibility gene. Lhr. BMC Evol. Biol. 11(1), 57, https://doi.org/10.1186/1471-2148-11-57 (2011).

4. Coyne, J. A. \& Orr, H. A. Speciation. (Sunderland, Massachusetts: Sinauer Associates (2004).

5. Haldane, J. Sex ration and unisexual sterility in animal hybrids. J. Genet. 12(2), 101-109, https://doi.org/10.1007/BF02983075 (1922).

6. Dobzhansky, T. Genetics and the Origin of Species. (New York, Columbia University Press (1937).

7. Muller, H. Isolating mechanisms. evolution, and temperature. Biol. Symp. 6, 71-125 (1942).

8. Presgraves, D. C. Sex chromosomes and speciation in Drosophila. Trends Genet. 24(7), 336-343, https://doi.org/10.1016/j. tig.2008.04.007 (2008).

9. Slotman, M., Della Torre, A. \& Powell, J. R. The genetics of inviability and male sterility in hybrids between Anopheles gambiae and An. arabiensis. Genetics 167(1), 275-287, https://doi.org/10.1534/genetics.167.1.275 (2004).

10. Turelli, M. \& Orr, H. A. The dominance theory of Haldane's rule. Genetics 140, 389-402, https://www.genetics.org/ content/140/1/389.short (1995)

11. Presgraves, D. C. Evaluating genomic signatures of "the large X-effect" during complex speciation. Mol. Ecol. 27(19), 3822-3830, https://doi.org/10.1111/mec.14777 (2018).

12. Lee, H. Y. et al. Incompatibility of nuclear and mitochondrial genomes causes hybrid sterility between two yeast species. Cell 135(6), 1065-1073, https://doi.org/10.1016/j.cell.2008.10.047 (2008).

13. Ting, C. T., Tsaur, S. C., Wu, M. L. \& Wu, C. I. A rapidly evolving homeobox at the site of a hybrid sterility gene. Science 282(5393), 1501-1504, https://doi.org/10.1126/science.282.5393.1501 (1998).

14. Bayes, J. J. \& Malik, H. S. Altered heterochromatin binding by a hybrid sterility protein in Drosophila sibling species. Science 326(5959), 1538-1541, https://doi.org/10.1126/science.1181756 (2009).

15. Liénard, M. A., Araripe, L. O. \& Hartl, D. L. Neighboring genes for DNA-binding proteins rescue male sterility in Drosophila hybrids. Proc. Natl. Acad. Sci. USA 113(29), E4200-E4207, https://doi.org/10.1073/pnas.1608337113 (2016).

16. Barbash, D. A., Siino, D. F., Tarone, A. M., \& Roote, J. A rapidly evolving MYB-related protein causes species isolation in Drosophila. Proc. Natl. Acad. Sci. USA 100(9), 5302-5307, https://doi.org/10.1073/pnas.0836927100 (2003).

17. Brideau, N. J. et al. Two Dobzhansky-Muller genes interact to cause hybrid lethality in Drosophila. Science 314(5803), 1292-1295, https://doi.org/10.1126/science.1133953 (2006). 
18. Phadnis, N. et al. An essential cell cycle regulation gene causes hybrid inviability in Drosophila. Science 350(6267), 1552-1555, https://doi.org/10.1126/science.aac7504 (2015).

19. Tang, S. \& Presgraves, D. C. Evolution of the Drosophila nuclear pore complex results in multiple hybrid incompatibilities. Science 323(5915), 779-782, https://doi.org/10.1126/science.1169123 (2009).

20. Presgraves, D. C., Balagopalan, L., Abmayr, S. M. \& Orr, H. A. Adaptive evolution drives divergence of a hybrid inviability gene between two species of Drosophila. Nature 423(6941), 715-719, https://doi.org/10.1038/nature01679 (2003).

21. Phadnis, N. \& Orr, H. A. A single gene causes both male sterility and segregation distortion in Drosophila hybrids. Science 323(5912), 376-379, https://doi.org/10.1126/science.1163934 (2009).

22. Cattani, M. V. \& Presgraves, D. C. Incompatibility between X chromosome factor and pericentric heterochromatic region causes lethality in hybrids between Drosophila melanogaster and its sibling species. Genetics 191(2), 549-559, https://doi.org/10.1534/ genetics.112.139683 (2012).

23. Sawamura, K., Yamamoto, M. T., \& Watanabe, T. K. Hybrid lethal systems in the Drosophila melanogaster species complex. II. The Zygotic hybrid rescue (Zhr) gene of D. melanogaster. Genetics 133(2), 307-313, https://www.genetics.org/content/133/2/307.short (1993).

24. Mihola, O., Trachtulec, Z., Vlcek, C., Schimenti, J. C. \& Forejt, J. A mouse speciation gene encodes a meiotic histone H3 methyltransferase. Science 323(5912), 373-375, https://doi.org/10.1126/science.1163601 (2009).

25. Bomblies, K. et al. Autoimmune response as a mechanism for a Dobzhansky-Muller-type incompatibility syndrome in plants. PLoS Biol. 5(9), e236, https://doi.org/10.1371/journal.pbio.0050236 (2007).

26. Larson, E. L., Keeble, S., Vanderpool, D., Dean, M. D. \& Good, J. M. The composite regulatory basis of the large X-effect in mouse speciation. Mol. Biol. Evol. 34(2), 282-295, https://doi.org/10.1093/molbev/msw243 (2016).

27. Ellegren, H. Genomic evidence for a large-Z effect. Proc. R. Soc. Lond. 276(1655), 361-366, https://doi.org/10.1098/rspb.2008.1135 (2009).

28. Dufresnes, C. et al. Empirical evidence for large X-effects in animals with undifferentiated sex chromosomes. Sci. Rep. 6(1), 1-7, https://doi.org/10.1038/srep21029 (2016).

29. Forejt, J. \& Iványi, P. Genetic studies on male sterility of hybrids between laboratory and wild mice (Mus musculus L.). Genet. Res. 24(2), 189-206, https://doi.org/10.1017/S0016672300015214 (1974).

30. Gregorová, S. et al. Sub-milliMorgan map of the proximal part of mouse Chromosome 17 including the hybrid sterility 1 gene. Mamm. Genome 7(2), 107, https://doi.org/10.1007/s003359900029 (1996).

31. Dzur-Gejdosova, M., Simecek, P., Gregorová, S., Bhattacharyya, T. \& Forejt, J. Dissecting the genetic architecture of $F_{1}$ hybrid sterility in house mice. Evolution. 66(11), 3321-3335, https://doi.org/10.1111/j.1558-5646.2012.01684.x (2012).

32. Bhattacharyya, T. et al. X chromosome control of meiotic chromosome synapsis in mouse inter-subspecific hybrids. PLoS Genet. 10(2), https://doi.org/10.1371/journal.pgen.1004088 (2014).

33. Forejt, J. Hybrid sterility in the mouse. Trends Genet. 12(10), 412-417, https://doi.org/10.1016/0168-9525(96)10040-8 (1996).

34. Forejt, J., Pialek, J. \& Trachtulec, Z. Hybrid male sterility genes in the mouse subspecific crosses. Evolution of the house mouse, (3), $482-503,(2012)$

35. Gregorová, S. \& Forejt, J. PWD/Ph and PWK/Ph inbred mouse strains of Mus m. musculus subspecies-a valuable resource of phenotypic variations and genomic polymorphisms. Folia Biol (Praha), 46(1), 31-41 https://fb.cuni.cz/volume-46-2000-no-1\#mus (2000).

36. Din, W. et al. Origin and radiation of the house mouse: clues from nuclear genes. J. Evol. Biol. 9(5), 519-539, https://doi.org/10.1046/ j.1420-9101.1996.9050519.x (1996).

37. Gregorová, S. et al. Mouse consomic strains: exploiting genetic divergence between Mus m. musculus and Mus m. domesticus subspecies. Genome Res. 18(3), 509-515, https://doi.org/10.1101/gr.7160508 (2008).

38. Baudat, F. et al. PRDM9 is a major determinant of meiotic recombination hotspots in humans and mice. Science 327(5967), 836-840, https://doi.org/10.1126/science.1183439 (2010).

39. Parvanov, E. D., Petkov, P. M. \& Paigen, K. Prdm 9 controls activation of mammalian recombination hotspots. Science 327(5967), 835-835, https://doi.org/10.1126/science.1181495 (2010).

40. Lustyk, D. et al. Genomic structure of Hstx2 modifier of Prdm9-dependent hybrid male sterility in mice. Genetics 213(2), https://doi. org/10.1534/genetics.119.302554 (2019).

41. Balcova, M. et al. Hybrid sterility locus on chromosome X controls meiotic recombination rate in mouse. PLoS Genet. 12(4), e1005906, https://doi.org/10.1371/journal.pgen.1005906 (2016).

42. Bhattacharyya, T. et al. Mechanistic basis of infertility of mouse intersubspecific hybrids. Proc. Natl. Acad. Sci. USA 110(6), E468-E477, https://doi.org/10.1073/pnas.1219126110 (2013).

43. Davies, B. et al. Re-engineering the zinc fingers of PRDM9 reverses hybrid sterility in mice. Nature 530(7589), 171, https://doi. org/10.1038/nature16931 (2016).

44. Smagulova, F., Brick, K., Pu, Y., Camerini-Otero, R. D. \& Petukhova, G. V. The evolutionary turnover of recombination hot spots contributes to speciation in mice. Genes Dev. 30(3), 266-280, https://doi.org/10.1101/gad.270009.115 (2016).

45. Hayashi, K., Yoshida, K. \& Matsui, Y. A histone H3 methyltransferase controls epigenetic events required for meiotic prophase. Nature 438(7066), 374, https://doi.org/10.1038/nature04112 (2005).

46. Mihola, O. et al. Histone methyltransferase PRDM9 is not essential for meiosis in male mice. Genome Res. gr-244426, https://doi. org/10.1101/gr.244426.118 (2019).

47. Von Deimlingi, O. H., Forejt, J. \& Wienker, T. F. Allelic profile at 37 biochemical loci of two inbred strains of the house mouse derived from wild Mus musculus musculus. Lab. Anim. 22(1), 61-66, doi:10.1258\%2F002367788780746610 (1988).

48. Dickinson, M. E. et al. High-throughput discovery of novel developmental phenotypes. Nature 537(7621), 508-514, https://doi. org/10.1038/nature19356 (2016).

49. Bakker, C. E. et al. Fmr1 knockout mice: a model to study fragile X mental retardation. Cell 78(1), 23-33, https://doi. org/10.1016/0092-8674(94)90569-X (1994).

50. Miyata, H. et al. Genome engineering uncovers 54 evolutionarily conserved and testis-enriched genes that are not required for male fertility in mice. Proc. Natl. Acad. Sci. USA 113(28), 7704-7710, https://doi.org/10.1073/pnas.1608458113 (2016).

51. Gu, Y. et al. Impaired conditioned fear and enhanced long-term potentiation in Fmr2 knock-out mice. J. Neurosci. 22(7), 2753-2763, https://doi.org/10.1523/JNEUROSCI.22-07-02753.2002 (2002).

52. Oliver, P. L. et al. Accelerated evolution of the $\operatorname{Prdm} 9$ speciation gene across diverse metazoan taxa. PLoS Genet. 5(12), e1000753, https://doi.org/10.1371/journal.pgen.1000753 (2009).

53. Ota, H. et al. Identification of the X-linked germ cell specific miRNAs (XmiRs) and their functions. PLoS one 14(2), e0211739, https://doi.org/10.1371/journal.pone.0211739 (2019).

54. Royo, H. et al. Silencing of X-linked microRNAs by meiotic sex chromosome inactivation. PLoS Genet. 11(10), e1005461, https:// doi.org/10.1371/journal.pgen.1005461 (2015).

55. Hoshino, Y. et al. Simple generation of hairless mice for in vivo imaging. Exp. Anim. 66(4), 437-445, https://doi.org/10.1538/ expanim.17-0049 (2017).

56. Sato, Y. et al. A mutation in transcription factor MAFB causes Focal Segmental Glomerulosclerosis with Duane Retraction Syndrome. Kidney Int. 94(2), 396-407, https://doi.org/10.1016/j.kint.2018.02.025 (2018). 


\section{Acknowledgements}

We thank the members of the Laboratory Animal Resource Center Sugiyama for helpful discussions and encouragement. This work was supported by Grants-in-Aid for Young Scientists (B) (17K14971: to S.M.), Scientific Research (B) (19H03142: to S.M.), and Scientific Research on Innovative Areas "Platform of Advanced Animal Model Support" (16H06276 to S.T., S.M., F.S.) from the Ministry of Education, Culture, Sports, Science, and Technology (MEXT). We would like to thank Editage (www.editage.com) for English language editing.

\section{Author contributions}

S.M., and F.S. were responsible for all experimental design. Y.D., Y.H., Ke.K., Ka.K., H.S. and S.T. developed KO mouse models. Ke.M. and K.N. performed genotyping and confirm KO phenotype. S.A., and A.Y. provided care for PWK mouse strain. Ka.M. and F.S. administered all animal experiments.

\section{Competing interests}

The authors declare no competing interests.

\section{Additional information}

Supplementary information is available for this paper at https://doi.org/10.1038/s41598-020-65986-y.

Correspondence and requests for materials should be addressed to S.M.

Reprints and permissions information is available at www.nature.com/reprints.

Publisher's note Springer Nature remains neutral with regard to jurisdictional claims in published maps and institutional affiliations.

(c) (i) Open Access This article is licensed under a Creative Commons Attribution 4.0 International License, which permits use, sharing, adaptation, distribution and reproduction in any medium or format, as long as you give appropriate credit to the original author(s) and the source, provide a link to the Creative Commons license, and indicate if changes were made. The images or other third party material in this article are included in the article's Creative Commons license, unless indicated otherwise in a credit line to the material. If material is not included in the article's Creative Commons license and your intended use is not permitted by statutory regulation or exceeds the permitted use, you will need to obtain permission directly from the copyright holder. To view a copy of this license, visit http://creativecommons.org/licenses/by/4.0/.

(C) The Author(s) 2020 